\title{
Ataques de cocodrilo de río (Crocodylus acutus) en Puerto Vallarta, J alisco, México: presentación de cinco casos
}

\section{American crocodile (Crocodylus acutus) attacks in Puerto Vallarta, Jalisco, Mexico: Presentation of five cases}

\section{Resumen}

Se presentan cinco casos de ataques por cocodrilos a personas, sin consecuencias fatales, en la región de Puerto VaIlarta, Jalisco, México (2007-2010). En cuatro de los casos, la víctima sufrió amputación del miembro superior o inferior, y en un caso sólo resultó con mordeduras avulsivas en el brazo. Con la documentación de estos cinco casos, el número de ataques por cocodrilo americano (Crocodylus acutus) en la costa del estado mexicano de Jalisco durante los últimos 52 años es de entre 30 y 31. Los resultados muestran un incremento en los ataques entre 0,57 y 0,59 por año. Se describen los casos y se proponen explicaciones sobre los posibles motivos de los ataques por parte de los cocodrilos.

Palabras clave: Ataque. Crocodylia. Mordedura. Conflicto humano-cocodrilo. Bahía de Banderas.

\section{Abstract}

Five cases of non-fatal crocodile attacks on people in the region of Puerto Vallarta, Jalisco, Mexico (2007-2010) are presented. In four cases the victim suffered amputation of upper or lower extremity, but only in one case the victim resulted in bitten on the arm. With the documentation of these five cases, the number of attacks by American crocodile (Crocodylus acutus) in the coast of the Mexican state of Jalisco for the past 52 years is between 30 and 31 . The results show an increase in crocodile attacks from 0,57 to 0,59 per year. We describe the cases and suggest possible explanations for the crocodile's attacks.

Key words: Attack. Crocodylia. Bite. Human-crocodile conflict. Banderas Bay.

\section{Introducción}

El registro más antiguo sobre la presencia del cocodrilo americano (Crocodylus acutus) en la Bahía de Banderas, región donde se encuentra la ciudad de Puerto Vallarta, Jalisco, México (Figura 1), se remonta al año 1861, fecha en la que un barco ballenero atracado en sus aguas documentó en su bitácora la ocurrencia y abundancia de los reptiles en algunas playas y en un río ${ }^{1}$. Pero en la década de los años 1930, de la contemplación se pasó a la explotación comercial de pieles para su exportación a diversas ciudades de los Estados Unidos y Alema$n^{2}{ }^{2}$. Con el inicio de la Segunda Guerra Mundial, los envíos a Alemania cesaron y la aplicación de leyes de prohibición por parte del gobierno mexicano (principalmente la veda decretada en 1970) $)^{3,4}$ terminaron con su aprovechamiento extensivo entre las décadas de los años 1940 y $1960^{2,5}$.

A partir de la década de los años sesenta del siglo pasado, la población humana de la región experimentó un crecimiento explosivo estrechamente asociado con fenómenos de afluencia masiva de turismo local y extranjero, contaminación de ríos y arroyos, expansión de la mancha urbana, entre otros $^{5}$. Precisamente, el avance del cerco urbano ha provocado que algunos hábitats naturales para los cocodrilos se vean reducidos o impactados ${ }^{6-8}$,
FG. Cupul-Magaña ${ }^{1}$

A. Rubio-Delgado ${ }^{2}$

C. Reyes-Núñez ${ }^{3}$

E. Torres-Campos ${ }^{3}$

LA. Solís-Pecero ${ }^{3}$

${ }^{1}$ Herpetólogo.

Universidad de

Guadalajara.

Puerto Vallarta, Jalisco,

México.

${ }^{2}$ Manejador de Vida

Silvestre. UMA

Acuetzpallin.

Puerto Vallarta, Jalisco, México.

${ }^{3}$ Bióloga. Proyecto COMACROM. Región

Pacífico Norte.

Puerto Vallarta, Jalisco, México.

Correspondencia: Dr. Fabio G. Cupul-Magaña Departamento de Ciencias Biológicas.

Centro Universitario de la Costa. Universidad de

Guadalajara.

Av. Universidad de Guadalajara №. 203, Delegación Ixtapa 48280 Puerto Vallarta, Jalisco, México.

Tel.: (322)2262218

Fecha de recepción: 20.JUN.2010

Fecha de aceptación: 29.JUN.2010 
lo que incrementa las posibilidades de que las personas y los reptiles entren en contacto y se generen situaciones de conflicto o ataques, principalmente en áreas urbanas cercanas a ríos, arroyos, esteros, charcas $^{9}$ y hasta trampas de agua de campos de golf. Por otra parte, se estima que la población de cocodrilos de diversas tallas dentro de la Bahía de Banderas (cálculo realizado al sumar los conteos máximos de animales obtenidos en diversos estudios conducidos en los esteros El Salado, Boca Negra, Boca de Tomates, El Quelele-Chino y trampas de agua de campos de golf (Figura 1)) es de 227 ejemplares $^{7,10-12}$.

Cuando los humanos y los cocodrilos entran en contacto, el ataque a los primeros posiblemente sea uno de los eventos resultantes ${ }^{13}$. Al respecto, se puede definir que un ataque ocurre desde el momento en que el cocodrilo realiza su primer avance o acercamiento a su presa, hasta el punto de matarla, devorarla o liberarla ${ }^{14}$. Para los aligatóridos de Norteamérica, se ha observado que las patofisiologías asociadas a sus ataques son amputación de extre-

\section{Figura 1. \\ Ubicación en México y localización de la mancha urbana de Puerto Vallarta, Jalisco, en la costa del océano Pacífico (Bahía de Banderas). Las flechas identificadas con los números 1 a 5 , señalan los sitios donde ocurrieron los cinco casos de ataques registrados entre 2007 y 2010. Las flechas identificadas con las letras a y b señalan los sitios donde ocurrieron los ataques a un niño de 8 años en 1993 y a una niña de 5 años en 2006, respectivamente. bn: Boca Negra; $c p$ : campo de golf Marina Vallarta; es: estero EI Salado; qe: El Quelele; t: Boca de Tomates.}

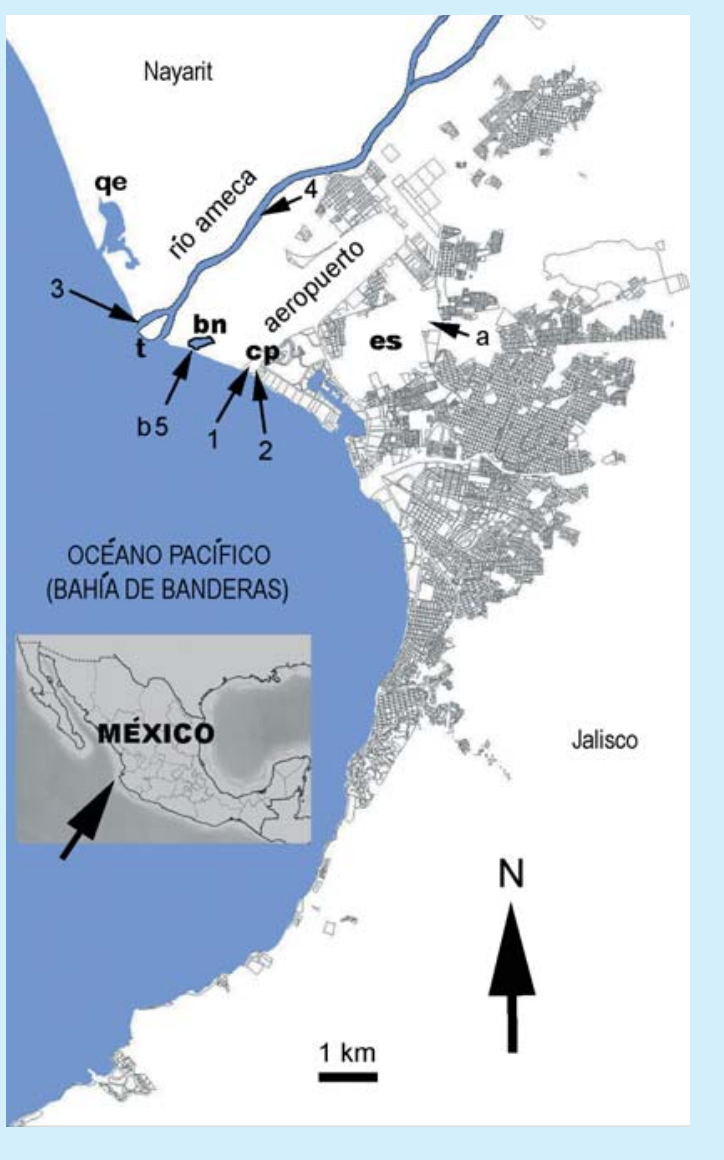

midades y aplastamiento de tejido y hueso, exsanguinación por amputación, marcas de mordedura (heridas por punción), así como septicemia generada por las mordeduras sépticas ${ }^{15}$. Estas mismas patofisiologías pueden ser reconocidas en los ataques del cocodrilo de río a víctimas humanas, aunadas a heridas avulsivas de bordes irregulares ${ }^{16}$ y marcas o impresiones de mordeduras que siguen el reconocible patrón largo y triangular de su hocico ${ }^{15}$.

Se ha establecido que las razones que explican la ocurrencia de ataques de cocodrilo, sin provocación de los humanos, responden a la defensa territorial (son territoriales y arremeten contra intrusos humanos u otros cocodrilos), defensa de nidos y/o crías (tienen cuidados parentales), caza para alimentación (son animales oportunistas que se alimentan de una amplia variedad de presas), confusión de identidad o humano como objetivo secundario (es posible que los cocodrilos dirijan su ataque a una mascota que puede ser un perro, a los peces que un pescador sujeta a su cintura o hasta al alimento que una persona le puede proporcionar deliberadamente $y$, al morder, equivoca su presa objetivo) y autodefensa (al tratar de atrapar o manipular cocodrilos, éstos pueden responder instintivamente con una mordedura para defenderse $)^{17}$.

Para un periodo de 49 años (1958-2007) se han documentado entre 25 y 26 ataques de cocodrilo en la costa de Jalisco, tres de ellos con consecuencias fatales, con una tendencia al incremento a partir de la década de los noventa ${ }^{18-20}$. El análisis de una muestra de 16 de estos casos, para el periodo de 1958 al 2002, arrojó que dos ataques ocurrieron durante la temporada reproductiva de la especie (cuando es más activa y agresiva); nueve en la temporada de eclosión de crías; en seis incidentes, la víctima realizaba faenas de pesca con el nivel del agua a la altura o por arriba de su cintura y, en tres de estos nueve eventos, el pescador llevaba sujeto el pescado al cuerpo ${ }^{18}$. Ponce-Campos ${ }^{19}$ argumenta que la recuperación de las poblaciones de cocodrilo en la región se relaciona directamente con el incremento de los conflictos entre hombre y cocodrilo; además, destaca que este riesgo aumenta por la pérdida de hábitat que experimenta la especie (reducción de su hábitat, uso de espacios urbanizados), por la ocurrencia de ejemplares de talla grande (tres o más metros), por condicionamiento forzado del cocodrilo al alimentarlo en espacios naturales (impronta), por temeridad y confianza de las personas al hacer uso de espacios donde habita el reptil, entre otros.

Con relación a lo anterior, Langley ${ }^{21}$ realizó una revisión de 567 casos de ataques del caimán americano (Alligator mississippiensis) en los Estados Unidos 
para los años de 1928 al 2008; encontró que al menos un cuarto de los ataques ocurrieron en sitios donde el caimán había sido alimentado previamente. Asimismo, este autor observó que un $39 \%$ de los conflictos fueron provocados por los humanos o perros. Por su parte, tanto Caldicott, et al. ${ }^{17}$ como Langley $^{21,22}$ proponen que el aumento en los conflictos entre el hombre y los crocodílidos es, en parte, producto del crecimiento de las poblaciones de los reptiles (que favorece el incremento en la probabilidad de encuentro con los humanos), las modificaciones a su hábitat, el aumento de la población humana y de las actividades recreacionales cerca del agua, entre otros.

En este trabajo se actualiza el registro de incidentes entre hombres y cocodrilos para la costa norte de Jalisco, México. Se presenta la información de cinco casos de ataques ocurridos entre el 2007 y el primer semestre de 2010.

\section{Material y métodos}

La información para documentar y estructurar los cinco casos de ataques ocurridos en Puerto Vallarta, Jalisco, y el límite político con el municipio vecino de Bahía de Banderas, Nayarit, se obtuvo de entrevistas directas realizadas a médicos y paramédicos que atendieron a las víctimas, a elementos de seguridad privada del campo de golf de Marina Vallarta y al personal de las dependencias de protección civil y policía municipal de ambas localidades, que estuvieron presentes en los sucesos y atendieron los llamados de emergencia. También, se entrevistó a las cinco personas atacadas. Asimismo, parte de la descripción de los cinco casos de ataque presentados aquí, se tomó de los informes no publicados ${ }^{23-26}$ de cuatro de los autores (ARD, CRN, ETC y LASP) de este artículo.

\section{Resultados}

Se presentan cinco casos de ataques de cocodrilo sobre humanos, ninguno con consecuencias fatales. Dos de los ataques ocurrieron en el campo de golf de Marina Vallarta, ubicado en la porción norte de la mancha urbana de Puerto Vallarta, Jalisco. Dos más en las aguas del río Ameca (límite interestatal entre Jalisco y Nayarit), a la altura de su desembocadura y del puente Ameca de la carretera federal no. 200. El último, en el estero Boca Negra, localizado aproximadamente a $300 \mathrm{~m}$ al noroeste del campo de golf de Marina Vallarta. En los dos primeros eventos, registrados el 2 de octubre de 2008 y el 3 de abril de
2010, las víctimas sufrieron amputaciones del miembro inferior izquierdo y superior izquierdo, respectivamente. Los conflictos tercero y cuarto sucedieron el 28 de septiembre de 2007 y el 13 de abril de 2008; en el tercer caso la víctima sufrió amputación de miembro superior izquierdo y, en el cuarto, sólo lesiones avulsivas en el bíceps del brazo izquierdo. En el quinto evento, el más reciente ocurrido el 5 de junio de 2010, la víctima sufrió fractura de ulna y radio y amputación de antebrazo izquierdo.

\section{Caso 1}

Un hombre de 39 años, residente de Puerto Vallarta, se encontraba con su pareja en el interior del campo de golf y a la orilla de una trampa de agua, alrededor de las 22:40 h. El sujeto ingresó sin permiso a la zona del campo de golf que es de acceso restringido. Según relata la víctima, con su pie izquierdo tocó el espejo de agua de la trampa (se asume que esto ocurrió mientras sostenía coito con su pareja), momento en el cual un cocodrilo de río o americano mayor a los dos metros de largo, lo sujetó del pie.

El cuerpo de bomberos municipal arribó en su auxilio y observó que el cocodrilo lo mantenía sujeto de la extremidad inferior izquierda, mientras se aferraba con sus brazos a un árbol cercano (al menos permaneció un par de horas en esta situación antes de recibir ayuda). Los bomberos le arrojaron una cuerda para rescatarlo (ya que parte de su cuerpo se encontraba dentro de la trampa de agua); sin embargo, cuando tensaron la cuerda para liberarlo, el cocodrilo realizó un giro sobre su propio cuerpo, conocido como "giro mortal", lo que le provocó politraumatismo en pantorrilla izquierda con cuatro fracturas expuestas de tipo espiral y compuesta de tibia y peroné, conminuta en astrágalo con partes de conexión de tejidos blandos, así como laceraciones mayores en los músculos gastrocnemio, sóleo y tibial anterior (Figura 2). Al rescatarlo y llevarlo al hospital, los médicos decidieron amputarle la extremidad izquierda por debajo de la rodilla.

\section{Caso 2}

Un joven de 15 años manifestó haber sido agredido por un cocodrilo. Se encontró de pie en estado de shock a las 01:10 h en las inmediaciones del campo de golf Marina Vallarta (la zona está limitada por una cerca perimetral de alambre de púas y tiene letreros informativos que prohíben ingresar a la zona privada y alertan sobre la presencia de cocodrilos). La víctima tenía amputado el brazo izquierdo, a la altura de la porción anterior del húmero. Fue asistido en primera instancia por elementos de la policía municipal y, posteriormente, recibió atención de socorristas de 
un hospital privado, quienes le aplicaron sedantes. Se le trasladó al nosocomio, donde se le práctico la amputación total del brazo izquierdo y se le administró como tratamiento de inicio 100 mg de Pentamin, 1 g de Amikasina, 2 g de Rosephin ${ }^{\circledR}$ y 500 mg de Histaline. Como tratamiento de mantenimiento, se le aplicó una ámpula de Pantosin cada 8 h, $1 \mathrm{~g}$ cada 24 h vía intravenosa de Amikasina, 30 mg cada 8 h vía intravenosa de Ketorolaco, una ámpula de Pantozol ${ }^{\circledR}$ cada 24 h vía intravenosa, una ámpula de Stadium ${ }^{\circledR}$ cada 8 h vía intravenosa, un ámpula de toxoide tetánico y una tableta de Gabapentina; además de solución Hartmann y solución salina $0.9 \%$ alternadas.

La víctima, residente de Guadalajara, Jalisco, fue entrevistada y relató no recordar lapsos del incidente. Sin embargo, a pesar de esto y de declararse también desorientada antes y durante el ataque, recuerda encontrarse dentro de una de las trampas de agua del campo de golf (cercana a la playa). En este espacio, fue arremetida varias veces a mordeduras y sacudidas (giros) por el cocodrilo (presentó heridas avulsivas en cara exterior e interior del muslo izquierdo y porción izquierda del pecho, así como escoriaciones leves en genitales). Comenta el joven que se golpeó repetidamente la cabeza con las rocas, además de sentir cómo el reptil le sujetaba de una pierna, mientras luchaba por mantenerse asido con su mano de las ramas cercanas (no recuerda si para

Figura 2 Víctima del caso 1 en sala de urgencias. Se observan las fracturas causadas en extremidad inferior izquierda por la torsión ejercida por el cocodrilo.

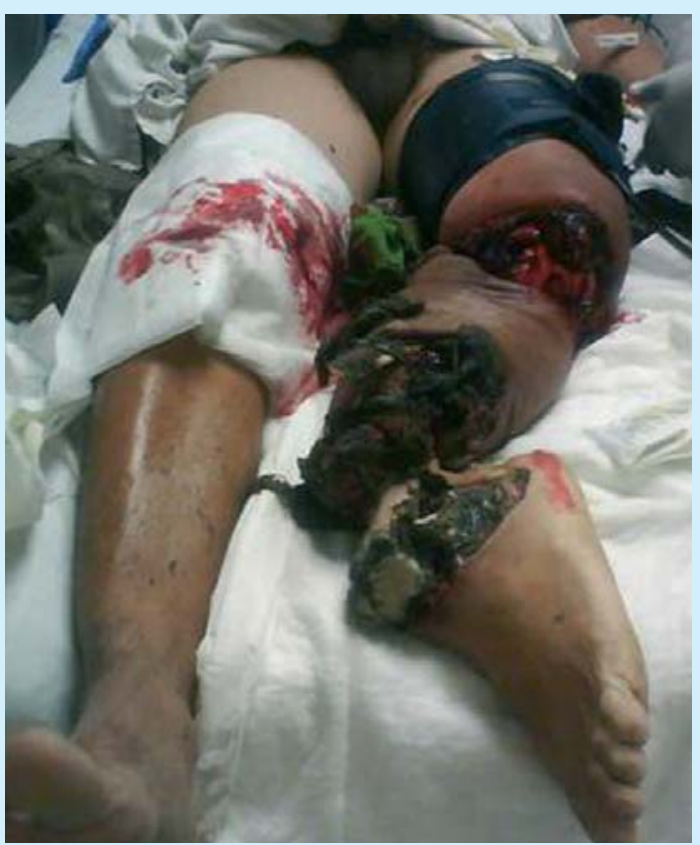

ese momento, ya había perdido su brazo). Una vez que el cocodrilo lo soltó, logró llegar a la orilla donde perdió el conocimiento en dos ocasiones. Ingresó de nuevo a la trampa de agua, hasta llegar al edificio de condominios aledaño al campo de golf donde fue asistido por los condóminos hasta la llegada de la policía y socorristas.

\section{Caso 3}

Un hombre de 34 años, residente de la delegación de Las Juntas en Puerto Vallarta, fue agredido por un cocodrilo cuando decidió ingresar a las aguas de la desembocadura del río Ameca para lavarse. Se sumergió hasta que el agua alcanzó el nivel de su cueIlo, en ese momento observó cómo un cocodrilo se le aproximó (a semejanza de un tronco en flotación) y abrió su hocico. En ese instante, el reptil le sujetó el brazo izquierdo, lo giró y desmembró a nivel del antebrazo. Nadó hacia la orilla, repeliendo los embates del cocodrilo, donde solicitó ayuda en las inmediaciones de un hotel de Nuevo Vallarta, Nayarit. La víctima fue llevada al hospital regional de Puerto Vallarta, donde se le realizó cirugía reconstructiva del miembro amputado en su segundo tercio.

Los tejidos amputados, depositados en una funeraria local, se revisaron. Se observó daño severo en la articulación húmero-cubital y radio-humeral, sin presencia de membrana y ligamento; el cóndilo humeral mostraba esquirlas y fractura en la tróclea; en el proceso de la apófisis coronoides del cúbito y, en cabeza y cuello del radio, se evidenció ligera separación de la articulación, quizás por la presión inversa o tirón que sufrió el brazo al encontrarse inmovilizado por el cocodrilo; en el cúbito y radio estaba ausente el ligamento interóseo del antebrazo y se advirtió una fractura oblicua transversa (pico de flauta) en sección media de ambos huesos, lo que aporta evidencia para asumir que el cocodrilo ejerció palanca para fracturar el hueso y giró para desmembrar el antebrazo. La porción distal del antebrazo y la mano no se recuperaron de la escena del ataque.

\section{Caso 4}

Un hombre de 42 años, residente de Puerto Vallarta, fue atacado por un cocodrilo en las aguas del río Ameca (a la altura del puente Ameca, donde existen letreros de advertencia sobre la presencia de cocodrilos) entre las 17:00 y 18:00 h. La víctima realizaba actividades recreativas con su familia (esposa y tres hijos) en la playa y márgenes del cuerpo de agua, cuando decidió realizar un clavado y nadar por debajo al agua. En esos momentos, sintió y observó cómo un cocodrilo jalaba de su brazo izquierdo. Con su brazo libre golpeó al reptil y logró liberarse. Fue 
auxiliado y trasladado a las instalaciones de protección civil de Bahía de Banderas, Nayarit, quien lo remitió a la Clínica no. 19 del IMSS en Mezcales para recibir atención, donde además se le detectó aliento alcohólico. Posteriormente, se trasladó a la Clínica no. 42 del IMSS en Puerto Vallarta, para su recuperación.

Se visitó a la víctima en el hospital para revisar sus lesiones. Se observó herida punzo-cortante de $22 \mathrm{~cm}$ de longitud en músculo bíceps y tríceps, con sangrado abundante con puntos de presión que no comprometieron al tejido óseo; así como varias heridas profundas en músculo braquiorradial y cabeza lateral del tríceps braquial, con compromiso en tendones, nervios y vasos sanguíneos (Figura 3 ). La herida avulsiva con trayectoria oblicua, localizada en la porción anterior del bíceps, se cree que fue ocasionada por el giro experimentado por el brazo al momento de liberarse del hocico del cocodrilo.

\section{Caso 5}

Un hombre de 74 años, residente de Puerto Vallarta, fue atacado por un cocodrilo de río a las 8:20 h del día 5 de junio de 2010 a la orilla del estero Boca Negra, mientras realizaba faenas de pesca con atarraya. La víctima relata que observó al cocodrilo "saltar" fuera del agua y, aunque intentó esquivarlo, fue atrapado del brazo izquierdo. Posteriormente, el reptil lo haló hacia el agua y lo hundió. Aunque el hombre logró liberarse, sufrió amputación por desgarre de antebrazo izquierdo desde el músculo braquiorradial hasta el tríceps y cabeza lateral del tríceps braquial, con exposición del cóndilo humeral de la articulación y un fragmento del cúbito hasta la tuberosidad radial (Figura 4). La persona fue trasladada al hospital donde se recuperó de sus lesiones. Cabe destacar que la víctima manifiesta haber golpeado los ojos del cocodrilo para lograr su liberación.

\section{Discusión}

Aunque en los estados mexicanos de Chiapas, Colima, Guerrero, Nayarit, Oaxaca, Quintana Roo, SinaIoa, San Luis Potosí, Tabasco y Tamaulipas ${ }^{19,27-30}$ se han registrado ataques de cocodrilo a personas, Jalisco es la única entidad del país que cuenta con una mayor cantidad de casos documentados y sistematizados ${ }^{18-20}$.

Los cinco ataques descritos se desarrollaron dentro o a orillas del agua, en sitios de acceso restringido por ser propiedad privada y/o zona de riesgo por presencia de cocodrilos. Por consiguiente, es posible que las víctimas, involuntariamente, provocaran la activación del mecanismo de ataque del reptil para la defensa del territorio (tres de los ataques ocurrieron en los meses de abril y junio, coincidentes con la temporada reproductiva, periodo de defensa territorial ante rivales o extraños por la presencia de nidos y crías) ${ }^{31}$, aunado al comportamiento de depredador oportunista ${ }^{32}$. Huerta-Ortega y Ponce-Campos ${ }^{18}$ encontraron que en los casos históricos de ataques de cocodrilo en la costa de Jalisco, el 87,5\% de los conflictos sucedieron dentro del agua. Al respecto, Caldicott, et al. ${ }^{17}$, comentan que el registro de los ataques del cocodrilo de agua salada australiano (Crocodylus porosus), del cocodrilo del Nilo (Crocodylus niloticus) y del caimán americano (Alligator mississippiensis), confirman que estos reptiles son más peligrosos dentro del agua que en la interface agua-tierra.

Los casos 1, 2, 3 y 5 ilustran el típico comportamiento de los crocodílidos y aligatóridos de tomar a una presa de gran tamaño de alguna extremidad para amputársela y causarle daños por aplastamiento en

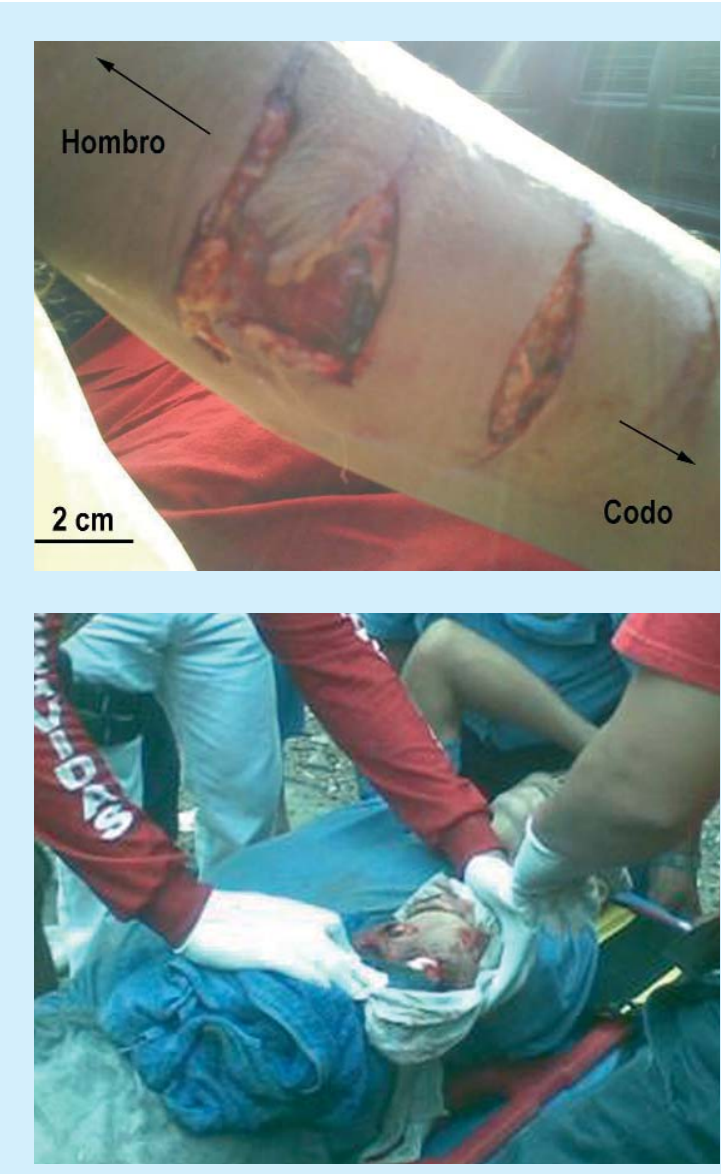

Figura 3 Acercamiento a la zona del bíceps del brazo izquierdo del adulto de 42 años del caso 4 , donde se aprecian las heridas por marcas de mordeduras.

Figura 4

Víctima del caso 5 atendida por paramédicos en el sitio del ataque. Se observa la amputación en el antebrazo izquierdo ocasionada por la mordedura del cocodrilo de río. 
huesos y tejidos suaves ${ }^{15,31}$. En el caso 4 la persona fue mordida en el brazo y logró liberarse, por lo que es posible que el cocodrilo sólo buscara ahuyentarla de su territorio o estaba en busca de presas más pequeñas ${ }^{31}$, ya que la víctima relató que se encontraba dentro del agua con uno de sus hijos pequeños (de nueve años de edad).

En el caso 5, posiblemente el cocodrilo se aproximó a la víctima en busca de comida, debido a que, según relatan testigos oculares, ese mismo día y en días previos, se observaron personas estimulando a los cocodrilos al arrojarles pescado. Curiosamente, cuando uno se acerca a esa laguna, al menos un cocodrilo de tamaño considerable se aproxima sin ningún temor, a escasos tres metros de la orilla. Tal vez, los cocodrilos estén improntados con los humanos al relacionarlos con la presencia de alimento de fácil $\operatorname{acceso}^{31,33}$.

En el caso 2 se documentó la utilización de antibióticos para el tratamiento del paciente que sufrió la amputación del brazo. Este protocolo es recomendable, debido a que se ha documentado que la mordedura del cocodrilo de río de la región de Puerto Vallarta es potencialmente séptica, en vista de que en su cavidad oral se han encontrado colonias bacterianas de Aeromonas hydrophila, Arizona sp, Citrobacter diversus, C. freundii, Enterococcus sp, Escherichia coli, Klebsiella pneumoniae, Neisseria sp, Pseudomonas sp y Streptococcus viridans ${ }^{34}$.

Se considera que los ataques de cocodrilo de río y caimanes americanos sobre los humanos son eventos raros o de muy baja frecuencia ${ }^{15,35}$. Para la costa de Jalisco, Ponce-Campos ${ }^{19}$ ha determinado, para el periodo de 1958 a 2002, que el promedio de conflictos por año es de 0,43, con un índice de 0,043 muertes por año. Sin embargo, si al registro previo de 25 o 26 ataques $^{20}$ se le adicionan los cinco casos registrados en este trabajo, entonces el promedio se incrementará a 0,57 o 0,59 ataques por año. Como la documentación de ataques realizada por HuertaOrtega y Ponce-Campos ${ }^{18}$ y Ponce-Campos ${ }^{19,20}$, ha sido exhaustiva y confiable, se asume que este índice es reflejo de un incremento en los casos de ataques, atribuibles, como se ha mencionado párrafos arriba, a un aumento en la población de reptiles, destrucción del hábitat y crecimiento del cerco urbano.

Asimismo, resulta interesante observar que los conflictos documentados en este trabajo se restringen exclusivamente a las trampas de agua del campo de golf de Marina Vallarta y a las inmediaciones del río Ameca (incluida su desembocadura conocida como Boca de Tomates) y estero Boca Negra. Pero, si con- sideramos los registros históricos de ataques en Puerto Vallarta, encontramos que es posible incluir otro espacio a esta lista de zonas conflictivas: el estero El Salado. En este cuerpo costero se registró un ataque no fatal a un niño de ocho años en 19936; asimismo, el estero Boca Negra, ya había sido escenario de un ataque no fatal a una niña de cinco años en $2006^{19}$. Con esta información, se puede proponer la elaboración de un mapa de zonas de riesgo de ataques por la presencia de cocodrilos (Figura 1).

En el caso del campo de golf es importante implementar mayores medidas de seguridad para evitar el ingreso de extraños (principalmente durante la noche). Al parecer, las medidas que establece la administración del campo de golf con los usuarios de sus servicios, como es la colocación de letreros que alertan sobre la presencia de cocodrilos en sus trampas de agua, han dado resultado, porque nunca se han suscitado conflictos entre éstos y los cocodrilos.

Para el estero El Salado, su declaración como Área Natural Protegida en el año 2000 ha hecho que los ataques, desde el registrado en $1993^{36}$, sean nulos porque el ingreso a la zona está totalmente restringido; además de que la mayor parte de su perímetro se encuentra limitado por una cerca. En cuanto al río Ameca, es importante un rediseño de la señalética existente para evitar la incidencia de ataques. Además, en este espacio, la alerta de presencia de cocodrilos no sólo debe ser en las cercanías de su desembocadura, sino que tendría que extenderse hasta $25 \mathrm{~km}$ río arriba, donde se han registrado cocodrilos y existen asentamientos humanos importantes ${ }^{7}$.

Langley ${ }^{21}$ enumera una serie de recomendaciones para evitar ser mordido por el caimán americano, las cuales son aplicables también para el cocodrilo de río o americano: no permitir que los niños se aproximen a los cuerpos de agua habitados por estos reptiles; no practicar la natación en zonas de riesgo y en aguas turbias; no alimentar a los animales con restos de comida dejados dentro o en la orilla del agua; evitar que las mascotas (perros) ingresen al agua donde no existe la seguridad de que se encuentre libre de estos reptiles; remover animales de espacios naturales si existe evidencia de que sean perniciosos; y no aceptarlos de mascotas. Además, en el caso de México, ante la existencia de un posible conflicto, acudir a las instancias de ecología municipal, protección civil, bomberos o los responsables del medio ambiente del estado o la federación, para que tomen cartas en el asunto. 


\section{Bibliografía}

1. Walker RC. Crocodiles in Puerto Vallarta. Homes \& Living. 2002;4:42-3.

2. Rodríguez R. Testimonio vallartense: Eduardo Güereña, plática con Roberto Rodríguez. México: El Colegio de Jalisco 1997.

3. Casas-Andreu G, Guzmán-Arroyo M. Estado actual sobre las investigaciones de cocodrilos mexicanos. $\mathrm{Bol}$ Inst Nal Invest Biol Pesqueras, Ser. Div. 1970;3:1-52.

4. Anónimo. Proyecto para la conservación, manejo y aprovechamiento sustentable de los Crocodylia en México (COMACROM). Instituto Nacional de Ecología. México, 2000. Disponible en: http://www.semarnat.gob.mx/gestionambiental/vidasilvestre/Docu ments/Preps/Prep_Coco.pdf

5. Guzmán-Mejía R, Anaya-Corona MC. Puerto VaIlarta: desde dónde vienes, hacia dónde vas. México: Universidad de Guadalajara 2009.

6. Gómez-Graciano FS, Cupul-Magaña FG. La técnica de análisis de amenazas como herramienta de diagnóstico ambiental: caso estero El Salado. Ciencia y Mar. 2001;5(14):33-42.

7. Cupul-Magaña FG, Hernández-Hurtado H, RubioDelgado A, García de Quevedo Machain R, González-Guevara L, Reyes-Juárez A. Conservación de un reptil prehistórico en la bahía de Banderas. Revista Mexicoa. 2001-2002;1-2:59-64.

8. Cupul-Magaña FG, Rubio-Delgado A, Reyes-Juárez A, de-Niz Villaseñor A. Historia natural del cocodrilo americano (Crocodylus acutus) en el estero Boca Negra, Jalisco, México: anidación y crecimiento de neonatos. Ciencia y Mar. 2004;8(23):31-42.

9. Gómez S, Cupul F. Estadísticas del programa de contingencias hombre-fauna silvestre. Boletín Nozootros. 2002;17:26-9.

10. Cupul-Magaña FG, Rubio-Delgado A, Reyes-Juárez A. American crocodile in Puerto Vallarta. Crocodile Spec Group Newsl. 2003;22(2):21-2.

11. Huerta-Ortega S. Dinámica poblacional del caimán (Crocodylus acutus, Cuvier, 1807, Crocodylidae) en Jalisco, México [Tesis de maestría]. Guadalajara: Universidad de Guadalajara 2005.

12. Hernández-Hurtado H, García de Quevedo Machain R, Hernández-Hurtado P. Los cocodrilos de la costa Pacífico Occidental (Michoacán, Colima y Jalisco) de México. En: Jiménez-Quiroz MC, Espino-Barr E. Los recursos pesqueros y acuícolas de Jalisco, Colima y Michoacán. México: Instituto Nacional de la Pesca 2006;375-89.

13. Anónimo. Human-wildlife conflict in Africa: causes, consequences and management strategies. FAO
Forestry Paper 157. Roma, 2009. Disponible en: http://www.fao.org/docrep/012/i1048e/i1048e00.pdf

14. Gruen RL. Crocodile attacks in Australia: challenges for injury prevention and trauma care. World J Surg. 2009;33:1554-61.

15. Harding BE, Wolf BC. Alligator attacks in southwest Florida. J Forensic Sc. 2006;51(3):674-7.

16. Mendieta C, Duarte A. Ataque por animales acuáticos (tiburón y cocodrilo). A propósito de dos casos fatales en la provincia de Bocas del Toro (Panamá). Cuad Med Forense. 2009;15(58):309-15.

17. Caldicott DGE, Cruser D, Manolis C, Webb G, Britton A. Crocodile attack in Australia: an analysis of its incidence and review of the pathology and management of crocodilian attacks in general. Wild Environ Med. 2005:16:143-59.

18. Huerta-Ortega S, Ponce-Campos P. Interacción hombre-cocodrilo en la costa de Jalisco, México. En: Proceedings of the Sixteen Working Meeting of Crocodile Specialist Group. Gland, Switzerland: IUCN 2002;200-3.

19. Ponce-Campos P. Un nuevo conflicto hombre-cocodrilo en Puerto Vallarta: análisis del caso, hipótesis, resultados y recomendaciones. Guadalajara, México: Bosque Tropical, AC. 2006.

20. Ponce-Campos P. Informe de la captura de un cocodrilo problema en Tomatlán, Jalisco, México. Guadalajara, México: Bosque Tropical, AC. 2007.

21. Langley RL. Adverse encounters with alligators in the United States: an update. Wild Environ Med. 2010;doi:10.1016/j.wem.2010.02.002.

22. Langley RL. Alligator attacks on humans in the United States. Wild Environ Med. 2005;16:119-24.

23. Reyes-Núñez C, Torres-Campos E, Solís-Pecero LA, Rubio-Delgado A. Informe a SEMARNAT, Conflicto Humano-Cocodrilo, Campo de Golf Marina VaIlarta, 2007. Informe no publicado. Puerto Vallarta, México, 2010.

24. Rubio-Delgado A, Reyes-Núñez C, Torres-Campos E, Solís-Pecero LA. Informe a DGVS, Conflicto Humano-Cocodrilo, Campo de Golf Marina Vallarta, 2010. Informe no publicado. Puerto Vallarta, México, 2010.

25. Solís-Pecero LA, Rubio-Delgado A, Reyes-Núñez C, Torres-Campos E. Informe a SEMARNAT, Conflicto Humano-Cocodrilo, desembocadura del Río Ameca, 2007. Informe no publicado. Nayarit, México, 2010.

26. Torres-Campos E, Solís-Pecero LA, Rubio-Delgado A, Reyes-Núñez C. Informe a SEMARNAT, Conflicto Humano-Cocodrilo, Ribera del Río Ameca, 2008. Informe no publicado. Nayarit, México, 2010. 
27. Alvarado D. Ataque de cocodrilos en el sur de Tamaulipas; temor cumplido. Disponible en: www. hoytamaulipas. net/?v1 = notas\&v2 $=43886 \&$ ti $\mathrm{t}=$ Ataque_de_cocodrilos_en_el_sur_de Tamaulipas; temor_cumplido (acceso 18.05.10).

28. Cocodrilo ataca a pescador en la Laguna de Mezcala. Disponible en: www.quadratin.com.mx/www1/noticia . $p h p ? i d=68469 \&$ seccion $=6$ (acceso 18.05.10).

29. Martínez de la Rosa A. Población creciente. Cocodrilos ya son un peligro en Colima. Diario de Colima. Disponible en: www.diariodecolima.com/opinion.php?var= 573 (acceso 18.05.10).

30. Ovando-Hidalgo N, Pérez-Sánchez E, RodríguezQuevedo F, Zequeira-Larios C, Macías-Valadez Treviño ME. Assessment of human-crocodile interaction in the state of Tabasco, Mexico. Crocodile Spec Group Newsl. 2008;27(4):10-1.
31. Pooley AC, Hines T, Shield J. Attacks on humans. En: Ross CA, Garnet S. Crocodiles and alligators. New York: Facts on File 1989;172-87.

32. Webb G, Manolis C. Australian crocodiles. Sydney: Redd New Holland 1998.

33. Alderton D. Crocodiles \& alligators of the world. London: Blandford 1999

34. Cupul-Magaña FG, Rubio-Delgado A, Reyes-Juárez A. La mordida del cocodrilo americano (Crocodylus acutus), ¿es potencialmente séptica? Rev Biomed. 2005; 16(1):65-7.

35. Kelly L. Crocodile: evolution's greatest survivor. Sydney: Allen \& Unwin 2006.

36. Navarro CJ, Navarro LE. Crocodylus acutus attack on Mexico's West coast. Crocodile Spec Group Newsl. 1995;14(2):20. 